\title{
Profiles of Tumor-Infiltrating Lymphocytes in a Case of Trichilemmal Carcinoma with Spontaneous Regression
}

\author{
Sadanori Furudate Taku Fujimura Akira Tsukada \\ Yukikazu Numata Yumi Kambayashi Setsuya Aiba \\ Department of Dermatology, Tohoku University Graduate School of Medicine, \\ Sendai, Japan
}

\section{Key Words}

Trichilemmal carcinoma $\cdot$ Spontaneous regression · Granulysin

\begin{abstract}
We describe the case of a 69-year-old Japanese patient with spontaneous regression of trichilemmal carcinoma. We investigated the immunohistochemical profiles of tumorinfiltrating lymphocytes, focusing on cytotoxic granules, granulysin-bearing cells and immunosuppressive cells, such as regulatory T cells and tumor-associated macrophages. Our present study describes some of the possible mechanisms of the self-regression of cutaneous malignant tumors and potential therapies for trichilemmal carcinoma by modifying the tumor microenvironment.
\end{abstract}

\section{Introduction}

Trichilemmal carcinoma (TLC) is a rare, cutaneous adnexal neoplasm that occurs on the sun-exposed areas of elderly people [1,2]. This neoplasm has been shown to exhibit outer root sheath differentiation and to be a malignant form of trichilemmoma. Cases with erythematous, tan, or flesh-colored plaques and nodules that may be keratotic or have superficial ulceration have been described. The lesions had usually been present for less than 1 year before the diagnosis and had a recent rapid growth phase. Though spontaneous regression of cutaneous tumors has been documented previously [3], that of TLC is extremely rare. In the present report, we describe a case of TLC with spontaneous regression and evaluate the profiles of the tumor-infiltrating lymphocytes (TILs). 


\section{Case Report}

A 69-year-old Japanese woman visited our outpatient clinic with a 3-year history of a tumor on her forehead. On her initial visit, physical examination revealed a papillomatous, dome-shaped, easy-tobleed nodule on her forehead (fig, 1a). The size of the tumor was of approximately $22 \times 16 \mathrm{~mm}$ in diameter. A biopsy specimen revealed cytologically atypical clear cells with abundant glycogen in the PAS staining. The tumor masses infiltrated the dermis with lobular growth patterns with keratinization, and partially with a horny cyst-like component (fig. 2a). According to the above findings, the patient was diagnosed of having TLC. Surprisingly, the tumor mass began decreasing 4 weeks after performing the skin biopsy (fig. 1a). The size of the tumor was of approximately $15 \times 15$ $\mathrm{mm}$ in diameter. We excised the lesion with a 1-cm surgical margin. Interestingly, the specimen from the widely excised area mainly consisted of dense infiltration of lymphocytes with collagen degeneration in the dermis, even around the hair follicles (fig. 2b). In the tumor-regressing area, atypical epitheloidal cells with atrophic changes were observed in the superficial dermis. According to the above findings, the patient was diagnosed with TLC with spontaneous regression.

To further investigate the possible mechanisms of the spontaneous regression, we employed immunohistochemical staining, especially focusing on the cytotoxic $\mathrm{T}$ cells and immunosuppressive lymphocytes as we have reported previously $[4,5]$. We performed immunohistochemical stainings in tumoral (fig. 3a, c, e) or regressive areas (fig. 3b, d, f) of the TLC for CD4, CD8, granulysin (fig. 3a, b), CD163 (fig. 3c, d), and Foxp3 (fig. 3e, f). The infiltrating mononuclear cells were mainly CD4 ${ }^{+}$, CD8 ${ }^{+}$ and CD163+ cells. Numerous Foxp3 ${ }^{\text {high }}$ cells were also scattered in the dermis of both the tumoral and regressing areas. Granulysin-bearing cells were detected mainly in CD8+ areas. We calculated the numbers of these TILs at the tumor-regressing and tumor-remaining areas. Concerning the effector cells, the numbers of CD8+ cells $(91.0 \pm 27.5$ vs. $151.4 \pm 35.2 \%)$ and granulysin-bearing cells $(25.4 \pm$ 13.5 vs. $56.4 \pm 16.6 \%$ ) were significantly increased in the tumor-regressing area compared to the tumor-remaining area. In contrast, there was no statistically significant difference between the numbers of immunosuppressive cells (Foxp3 ${ }^{+}$regulatory T cells: $28.4 \pm 8.0$ vs. $46.8 \pm 15.6 \%$, CD $163^{+}$ M2 macrophages $54.0 \pm 7.7$ vs. $102.0 \pm 41.0 \%$ ). The numbers of immunoreactive cells were counted using an ocular grid of $1 \mathrm{~cm}^{2}$ at a magnification of $400 \times$, and are summarized in fig. 4 . Staining of infiltrated lymphocytes was examined in more than five random, representative fields from each section. For a single comparison of two groups, Student's t test was used. The level of significance was set at $\mathrm{p}=0.05$.

\section{Discussion}

TLC is a rare, cutaneous adnexal neoplasm that occurs on the sun-exposed areas of elderly people [1, 2]. In a rare case, Garrett et al. [6] reported TLC occurring in a renal transplant patient. This report suggested the correlation between immunosuppressive circumstances and the carcinogenesis of TLC. Interestingly, in our case, histological findings revealed spontaneous regression of the tumor with dense infiltration of lymphocytes around the tumor, which might suggest the induction of an anti-tumor immune response against TLC.

Specific activation of the immune system to control cancer growth has been a longstanding goal in cancer immunology and medical oncology. We have previously reported possible therapies for melanoma in which an anti-tumor immune response is induced by intratumoral administration of polyinosinic-polycytidylic acid/cationic liposome, which suggested the possibility of intra-tumoral administration of immunomodulating reagents for the treatment of skin cancers [7]. More recently, we have also described the immunomodulating effects of peritumoral injection of interferon beta, which might suggest a relationship between the tumor regression and the profiles of the TILs [8]. Therefore, in the present case, we examined the profiles of 
tumor-infiltrating cells in TLC to investigate the possible mechanisms of the spontaneous regression of the cutaneous tumor.

Concerning cutaneous tumors, particularly melanoma, there is evidence suggesting that the immune system plays a central role in mediating tumor regression [3]. We have previously described a case of basal cell carcinoma with spontaneous regression and assessed the profiles of TILs, which revealed an increase in effector T cells and a decrease in immunosuppressive cells, including regulatory T cells and CD163+ M2 macrophages in the tumor-regressing area [4]. However, to our knowledge, there is no report on TLC with spontaneous regression or on mechanisms underlying the spontaneous regression of TLC. In our present case, although increased numbers of $\mathrm{CD} 163^{+} \mathrm{M} 2$ macrophages and Foxp3 ${ }^{+}$Tregs were observed in both tumor-remaining and tumor-regressing areas, the numbers of effector cytotoxic T cells were significantly increased in the tumor-regressing area. As previous reports suggested, the expression of granulysin correlated with the prognosis of cancer patients $[9,10]$. Altogether, the spontaneous regression of TLC might be related to the increased numbers of granulysin-bearing cells and CD8+ cells.

In summary, we described a case of TLC with spontaneous regression. Our present study sheds light on the immunological mechanisms of tumor rejection in human skin cancer and might suggest a possible therapy for such skin tumors by modifying the tumor microenvironment.
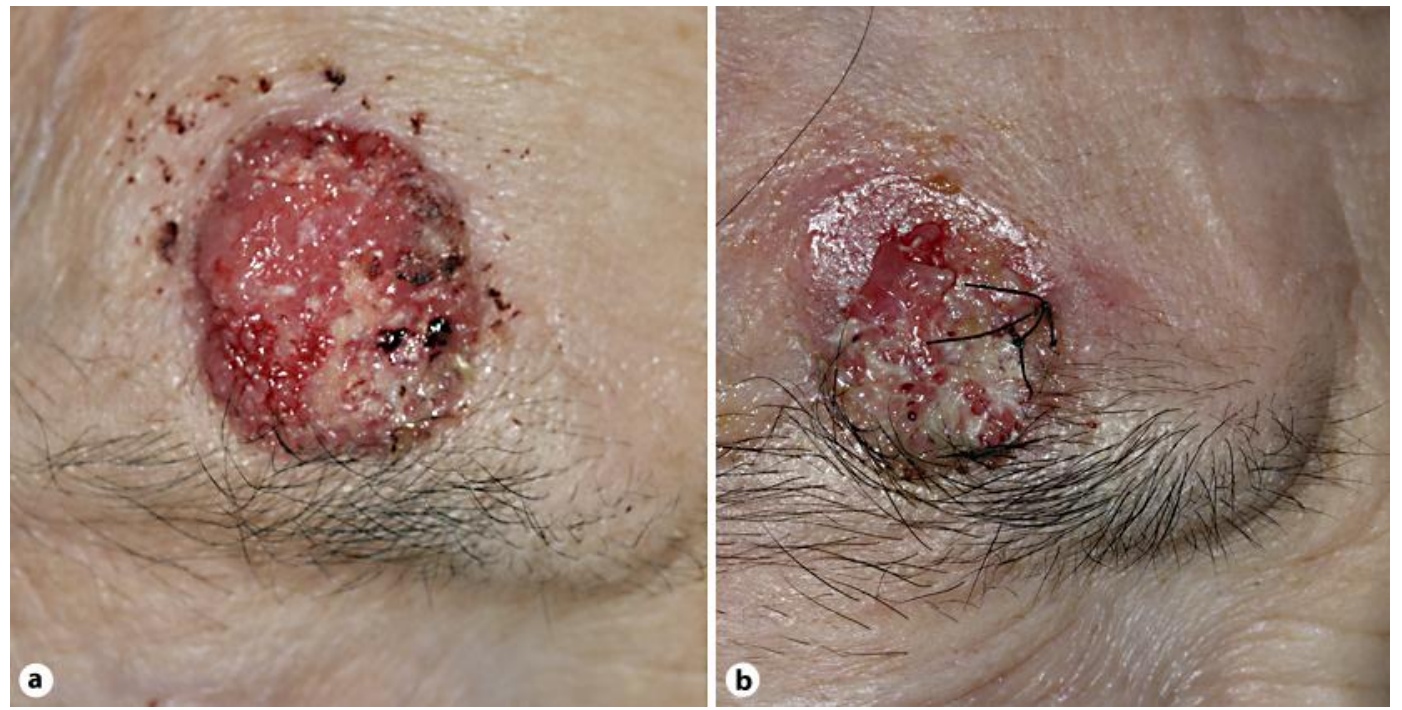

Fig. 1. a Papillomatous, dome-shaped, pinkish tumor on the forehead with erosion. b Four weeks after the skin biopsy. 

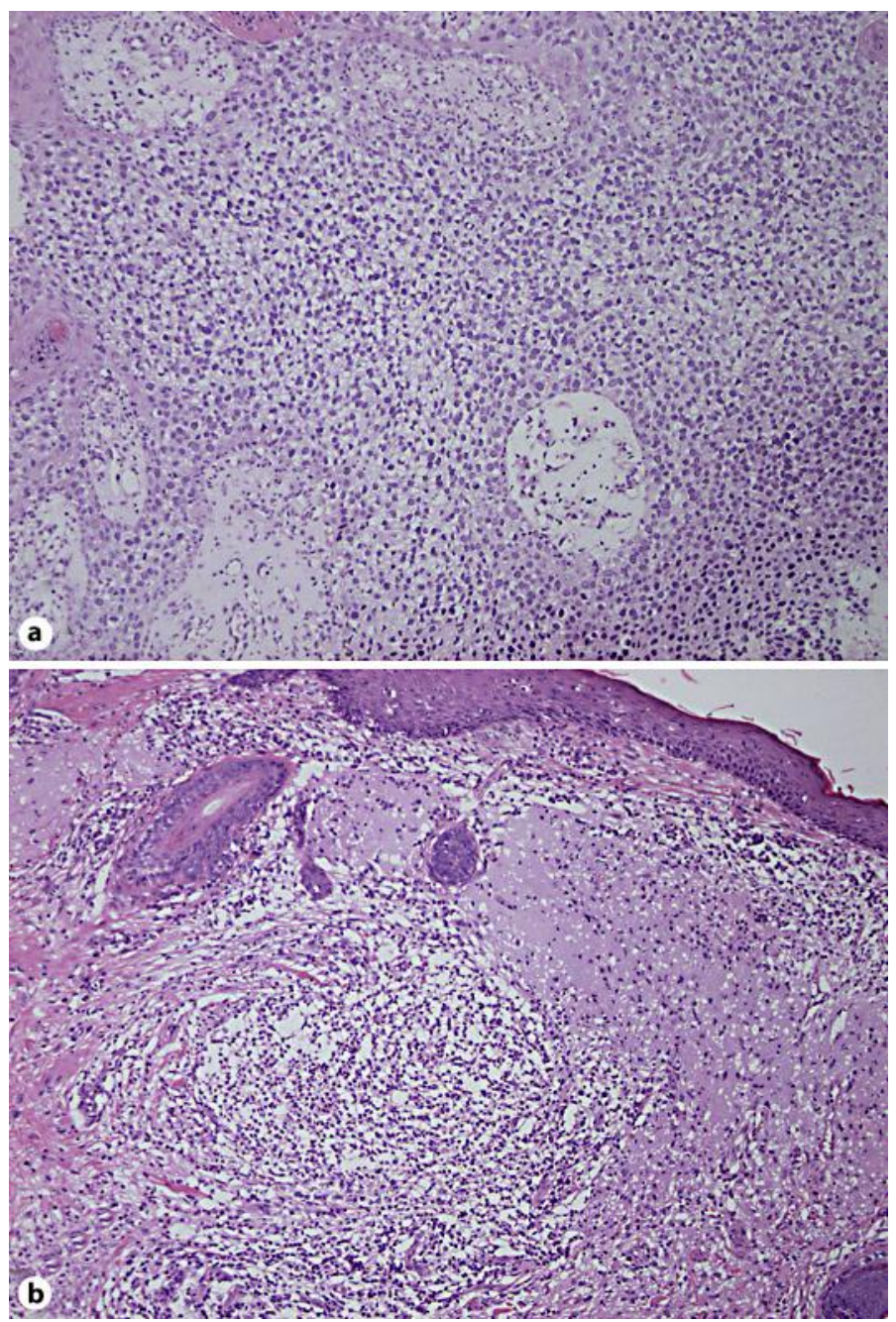

Fig. 2. Biopsy specimen (tumor-remaining area). a The tumor masses infiltrated the dermis with lobular growth patterns with keratinization, and partially with a horny cyst-like component. b Tumor regression area: dense infiltration of lymphocytes with collagen degeneration in the dermis, even around the hair follicles. Original magnification $100 \times$. 

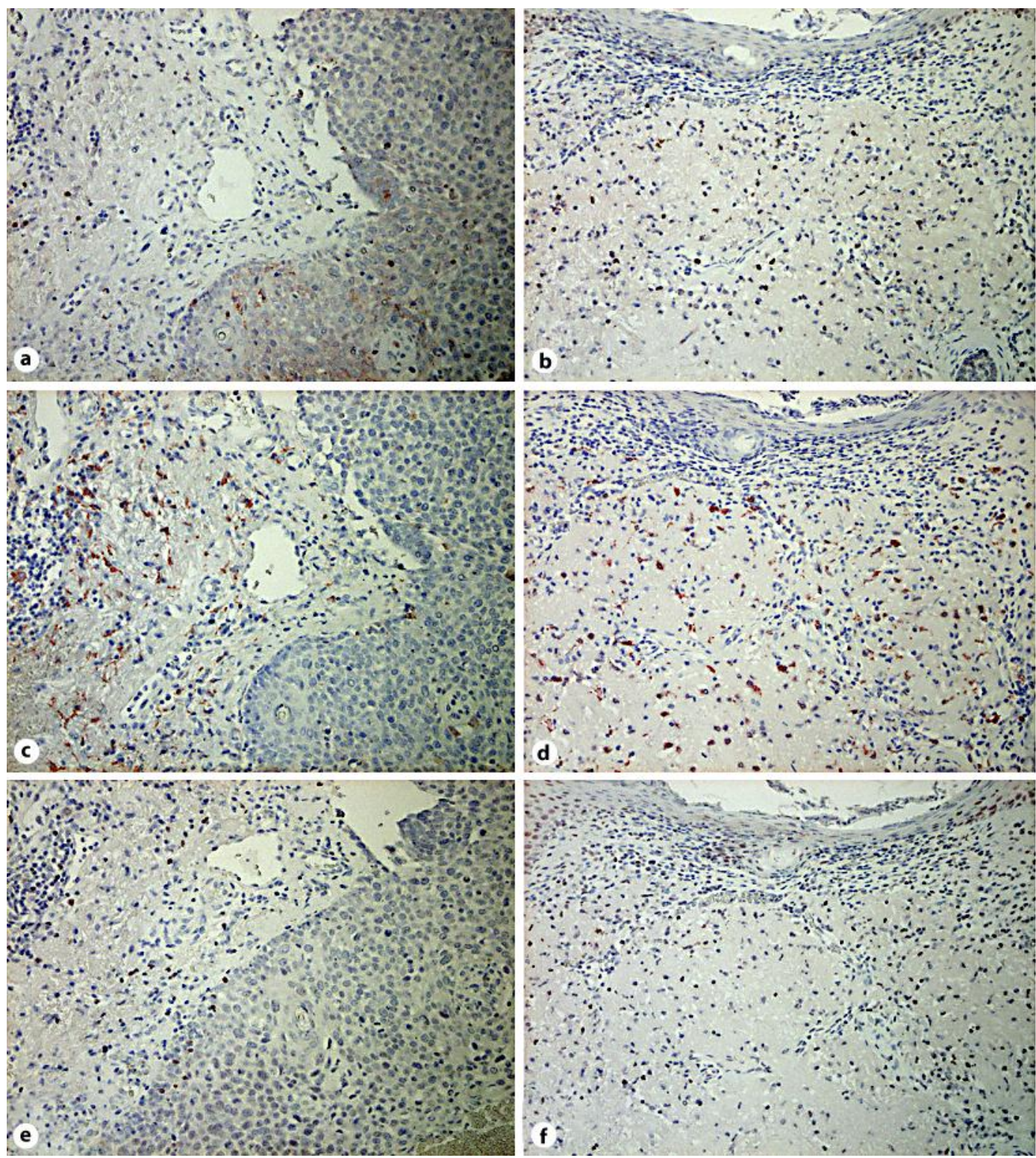

Fig. 3. Paraffin-embedded tissue samples from the patient were stained as follows: the sections were developed with new fuchsin for granulysin (a, b), CD163 (c, d), and Foxp3 (e, f). Tumoral areas: a, c, e. Regression areas: b, d, f. Original magnification $200 \times$. 


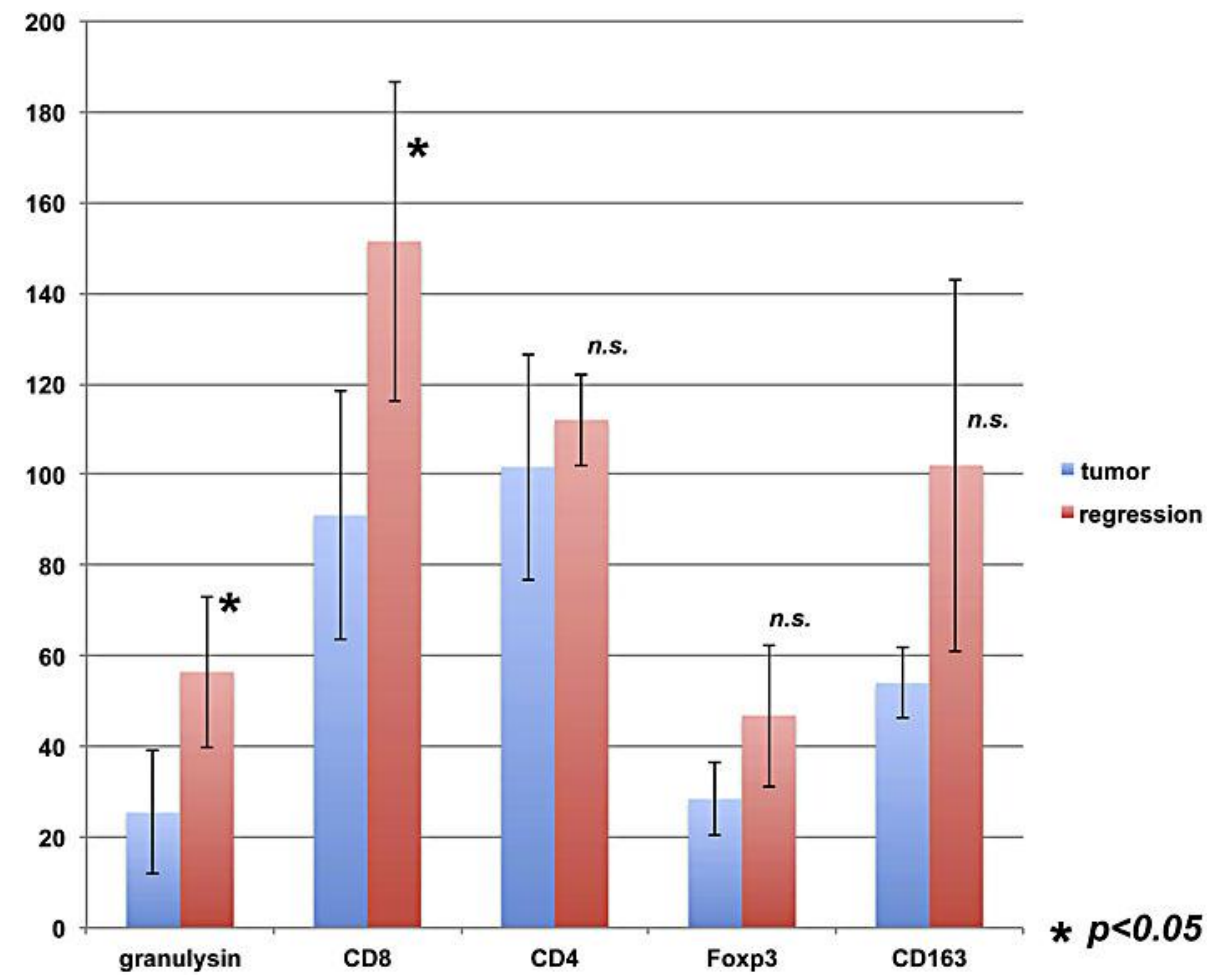

Fig. 4. Summary of the numbers of immunoreactive cells in the regressive or tumoral area of the TLC. Five representative fields of each section were selected from both the epidermis and the dermis, which showed a dense lymphoid infiltrate. The number of immunoreactive cells was counted using an ocular grid of $1 \mathrm{~cm}^{2}$ at a magnification of $400 \times$. Data are expressed as the means \pm SD of the numbers in each area. ${ }^{*} \mathrm{p}<0.05$. 


\section{References}

1 Reis JP, Tellechea 0, Cunha MF, Baptista AP: Trichilemmal carcinoma: review of 8 cases. J Cutan Pathol 1993;20:44-49.

-2 Garrett AB, Azmi FH, Ogburia KS: Trichilemmal carcinoma: a rare cutaneous malignancy: a report of two cases. Dermatol Surg 2004;30:113-115.

3 Hunt MJ, Halliday GM, Weedon D, Cooke BE, Barnetson RS: Regression in basal cell carcinoma: an immunohistochemical analysis. Br J Dermatol 1994;130:1-8.

-4 Fujimura T, Kakizaki A, Kambayashi Y, Aiba S: Basal cell carcinoma with spontaneous regression: a case report and immunohistochemical study. Case Rep Dermatol 2012;4:125-132.

-5 Fujimura T, Itoigawa A, Haga T, Aiba S: Cutaneous lymphadenoma: a case report and immunohistochemical study. Case Rep Dermatol 2012;4:50-55.

-6 Garrett AB, Scott KA: Trichilemmal carcinoma: a case report of a rare skin cancer occurring in a renal transplant patient. Transplantation 2003;76:1131.

-7 Fujimura T, Nakagawa S, Ohtani T, Ito Y, Aiba S: Inhibitory effect of the polyinosinic-polycytidylic acid/cationic liposome on the progression of murine B16F10 melanoma. Eur J Immunol 2006;36:33713380.

-8 Fujimura T, Okuyama R, Ohtani T, Ito Y, Haga T, Hashimoto A, Aiba S: Perilesional treatment of metastatic melanoma with interferon-beta. Clin Exp Dermatol 2009;34:793-799.

-9 Pagès F, Berger A, Camus M, Sanchez-Cabo F, Costes A, Molidor R, Mlecnik B, Kirilovsky A, Nilsson M, Damotte D, Meatchi T, Bruneval P, Cugnenc PH, Trajanoski Z, Fridman WH, Galon J: Effector memory T cells, early metastasis, and survival in colorectal cancer. N Engl J Med 2005;353:2654-2666.

10 Kakizaki A, Fujimura T, Mizuashi M, Watabe A, Kambayashi Y, Aiba S: Successful treatment of syringotropic CD8+ mycosis fungoides accompanied by hypohidrosis with vorinostat and retinoid. Australas J Dermatol 2012, E-pub ahead of print. 